\title{
THE OUTDOOR RAPID CALIBRATION TECHNIQUE AND REALIZATION OF NON- METRIC DIGITAL CAMERA BASED ON THE METHOD OF MULTI-IMAGE DLT AND RESECTION
}

\author{
ZHANG Qiang ${ }^{\mathrm{a}}$, SHEN Jian-jing ${ }^{\mathrm{b}}$, SUN Meng-qing ${ }^{\mathrm{c}}$ \\ a Institute of Surveying and Mapping, Zhengzhou Henan 450052, China, ZQ_28@126.com \\ ${ }^{\mathrm{b}}$ Institute of Surveying and Mapping, Zhengzhou Henan 450052, China, shenjj@vip.sina.com \\ c North China University of Water Resources and Electric Power, Zhengzhou Henan 450046, China, mqsun00@126.com
}

Commission I, WG I/3

KEY WORDS: Non-Metric Camera, Calibration, Multi-Image DLT, Multi-Image Resection

\begin{abstract}
:
For non-metric CCD digital camera features and the needs of Rapid field non-metric cameras calibration, the error sources was detailed analyzed and a mathematical calibration model has been founded. Both detailed multi-image group iterative method for solving DLT coefficient, the elements of interior orientation and distortion parameters of lens and the multi-image resection method for solving the elements of interior orientation, elements of exterior orientation and distortion parameters of lens have been discussed. A standard steel cage (e.g. Figure 1) has been made for real calibrating non-metric cameras outdoor quickly. In order to verify the accuracy, each method mentioned has been used to solve elements of interior orientation and distortion parameters with the same camera (e.g. Figure 2) and the same test images. The results of accuracy show that the maximum X error was $0.2585 \mathrm{~mm}$, the maximum Y error was $0.6719 \mathrm{~mm}$ and the maximum $\mathrm{Z}$ error was $0.1319 \mathrm{~mm}$ by using multi-image DLT algorithm. On the other hand, the maximum $\mathrm{X}$ error was $0.1914 \mathrm{~mm}$, the maximum $\mathrm{Y}$ error was $0.9808 \mathrm{~mm}$ and the maximum $\mathrm{Z}$ error was $0.1453 \mathrm{~mm}$ by using multi-image resection algorithm. The forward intersection accuracy of the two methods was quite, and the both were less than $1 \mathrm{~mm}$. By using multi-image DLT algorithm the planimetric accuracy was less than $0.2585 \mathrm{~mm}$ and the height accuracy was less than $0.6719 \mathrm{~mm}$. On the other hand, by using multi-image resection algorithm the planimetric accuracy was less than $0.1914 \mathrm{~mm}$ and the height accuracy was less than $0.9808 \mathrm{~mm}$. The planimetric accuracy of resection algorithm was the better than DLT algorithm, but the elevation accuracy of DLT algorithm was the better than resection algorithm. In summary both method can be accepted for nonmetric camera calibration. But also the solver accuracy in the inner orientation elements and distortion parameters was not very high has been noted. However for non-metric camera, the true value of inner orientation elements and lens distortion were unknown did not affect the accuracy of photogrammetry.
\end{abstract}

\section{INTRODUCTION}

The digital camera is based on the Semiconductor Technology, The Charge Coupled Devices (CCD), which are arrange in a line or a rectangular region square, constitute the imagesensitive linear array or the imagesensitive surface, auxiliary by optical system, mechanical device and electronic circuit's equipment. The digital CCD camera has the volume small, facile, durable, the actual maintenance convenience as well as merits and so on strong earthquake resistance ability. More importantly, speaking of the digital CCD sensor's character and the sensitivity, it can be stability for a long time. This means that the digital CCD camera may be calibrated(Wang Lei, 2002). The GDOP of digital CCD camera's focal plane decides in these photoconductive cells's geometry position precision. Each photoconductive cell is equal to a pixel, it records a single color value, forms a picture element, and all picture elements form an image. A digital CCD camera's big advantage is each element's position does not change. Therefore it does not need to use the collimation marks to determine the CCD camera's internal relevant position. Otherwise the film is not so, it has the film distortion error.

There are two types digital cameras can be use for photogrammetry: metric digital camera and non-metric digital camera. With the rapid development of science and technology, the performance of normal non-metric digital camera is increasingly enhanced, which easy satisfies many photographic conditions with a low cost. Therefore, the digital camera is used to the close-range photogrammetry widely. But in generally speaking, the elements of interior orientation are unknown and the lens distortion is too bigger for normal non-metric digital camera. These shortcomings badly influence measurement accuracies. Recently the research of non-metric digital camera calibration was more and more.

In the literature that I have seen(HE Min, 2011a)(Miao Hongjie, 2005), in the digital camera calibration, which generally is based on outdoor standardization field or indoor standardization field, we can obtain elements of interior orientation and/or the lens distortion by solute the resection equation or direct linear transformation (DLT) equation. When we solute interior orientation parameters and lens distortion parameters simultaneously in three dimension with multi-Images, we find it difficult.

In this paper, we has founded two mathematical model of the camera calibration, put forward the adoption of the multiimages iteration method to calculate the coefficients of the DLT/resection and distortion parameters in groups by threedimensional ground control points (GCP), The precision of camera calibration are confirmed by the experiments of multiimage resection and forward intersection, and the satisfactory effect is obtained.

\section{ANALYSIS OF ERROR SOURCES}

The errors which the digital CCD camera produces are mainly divided into the spectrum error and the geometrical error. The spectrum error, which is mainly caused by CCD, consists of 
background noise, accidental noise and invalid pixel. The geometrical error mainly is produces by its optics lens and the image sampling circuit. The geometrical error divides into optics error and the electricity error generally. Then we introduced separately the CCD camera produces spectrum error and geometrical error's analysis and examination.

\subsection{Spectrum Error}

2.1.1 Determination and Elimination of Background Noise: When the camera lens cap is close and the f-number is 22, we release the shutter and get a gray matrix from the photo. This matrix is called background noise. Background noise can affect signal-noise ratio of the digital image. A good camera and the sampling system should have a low amplitude and uniform background noise. The way of eliminating the background noise is to use current image to subtract its background noise.

2.1.2 Elimination of Accidental Noise: The accidental noise is presents the random along with the time change gradation step change, when carries on the examination, may absorb many phantoms continuously to the identical scenery. Takes its gradation average value to take the final grey level, like this may eliminate the accidental noise effectively the influence.

2.1.3 Elimination of Invalid Pixel: The CCD maybe exist individual damage cell or the cell of physical character is unstable. This kind of cell is called invalid pixel. The way of Elimination is to interpolate this invalid pixel by the neighbouring pixel

\subsection{Geometrical Error}

The geometrical error of the digital CCD camera mainly refers to the lens distortion error. It includes the radial distortion error and the tangential distortion error. The lens distortion error generally performs for the centre small and peripheral big. The radial distortion error is

$$
\left\{\begin{array}{l}
\Delta x=\left(x-x_{0}\right)\left(k_{1} r^{2}+k_{2} r^{4}+k_{3} r^{6}+\cdots\right) \\
\Delta y=\left(y-y_{0}\right)\left(k_{1} r^{2}+k_{2} r^{4}+k_{3} r^{6}+\cdots\right)
\end{array}\right.
$$

The tangential distortion error (Shao Xihui, 1991) is

$$
\left\{\begin{array}{l}
\Delta x=p_{1}\left[r^{2}+2\left(x-x_{0}\right)^{2}\right]+2 p_{2}\left(x-x_{0}\right)\left(y-y_{0}\right) \\
\Delta y=p_{2}\left[r^{2}+2\left(y-y_{0}\right)^{2}\right]+2 p_{1}\left(x-x_{0}\right)\left(y-y_{0}\right)
\end{array}\right.
$$

where

$$
\begin{aligned}
& r=\sqrt{\left(x-x_{0}\right)^{2}+\left(y-y_{0}\right)^{2}} \\
& x, y=\text { pixel coordinates } \\
& x_{0}, y_{0}=\text { principal point of photograph coordinates } \\
& k_{1}, k_{2}, k_{3} \ldots=\text { radial distortion coefficients } \\
& p_{1}, p_{2},=\text { tangential distortion coefficients }
\end{aligned}
$$

\section{CALIBRATION ALGORITHM OF MULTI-IMAGE DLT}

The indoor specialized optics check-out facility and the outdoor standardization field are two general methods of calibration. However, this paper has used a standard portable steel cage outdoor, which is approximately $50 \mathrm{~cm}$ long, $50 \mathrm{~cm}$ wide and $50 \mathrm{~cm}$ high. It has six levels of elevation differences altogether approximately $50 \mathrm{~cm}$ and altogether 61 control points. This site is presented in Figure 1.

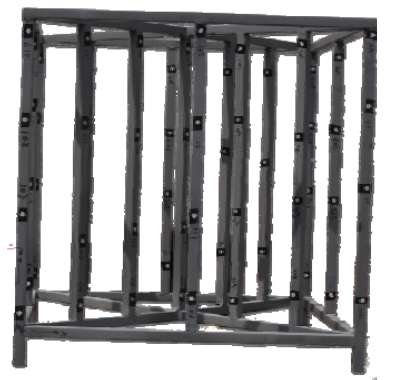

Figure 1 Standard Portable Steel Cage

For the non-metric digital camera, the principal-distance $\mathrm{f}$ is unknown and it has not collimating marks. Therefore DLT, which founds the relationship between the photo coordinate system and the ground coordinate system, is direct algorithm without the elements of interior orientation. It is very fit for the calibration of digital camera. However, the traditional DLT is low precision and not easy to converge. So solution is to be difficult. When the control points have small altitude difference, we can not synchronously obtain elements of interior orientation and the lens distortion by solute the DLT equation. So we put forward the adoption of the multi-images iteration method to calculate the coefficients of the DLT and distortion parameters in groups by three-dimensional ground control points (GCP). The precision of camera calibration are confirmed by the experiments of multi-image resection and forward intersection, and the satisfactory effect is obtained.

\subsection{Improved DLT with Distortion Error Correction}

The DLT equation with distortion error correction is

$$
\left\{\begin{array}{l}
x-x_{0}+\Delta x+\frac{X L_{1}+Y L_{2}+Z L_{3}+L_{4}}{X L_{9}+Y L_{10}+Z L_{11}+1}=0 \\
y-y_{0}+\Delta y+\frac{X L_{5}+Y L_{6}+Z L_{7}+L_{8}}{X L_{9}+Y L_{10}+Z L_{11}+1}=0
\end{array}\right.
$$

where $\quad X, Y, Z=$ GCP coordinates

$L_{1}, L_{2}, L_{3} \ldots=$ DLT coefficients

$\triangle x, \triangle y=$ distortion coefficients

It is obvious that we can calculate the DLT coefficients and the distortion parameters with some control points from Eq. (3). If we ignore high order terms of Eq. (1), the distortion parameters are $k_{1}, k_{2}, k_{3}, p_{1}, p_{2}$. We need 8 control points at least to solve the 11 DLT coefficients and 5 distortion parameters.

\subsection{Solution of the DLT Coefficients}

Observation equation is

$$
\left\{\begin{array}{l}
-\left(L_{1} X+L_{2} Y+L_{3} Z+L_{4}\right) / A-L_{9} X\left(x^{\prime}+\Delta x\right) / A \\
-L_{10} Y\left(x^{\prime}+\Delta x\right) / A-L_{11} Z\left(x^{\prime}+\Delta x\right) / A-\left(x^{\prime}+\Delta x\right) / A=v_{x} \\
-\left(L_{5} X+L_{6} Y+L_{7} Z+L_{8}\right) / A-L_{9} X\left(y^{\prime}+\Delta y\right) / A \\
-L_{10} Y\left(y^{\prime}+\Delta y\right) / A-L_{11} Z\left(y^{\prime}+\Delta y\right) / A-\left(y^{\prime}+\Delta y\right) / A=v_{y}
\end{array}\right.
$$

Type of Matrix is

$$
V=C \Delta+L
$$




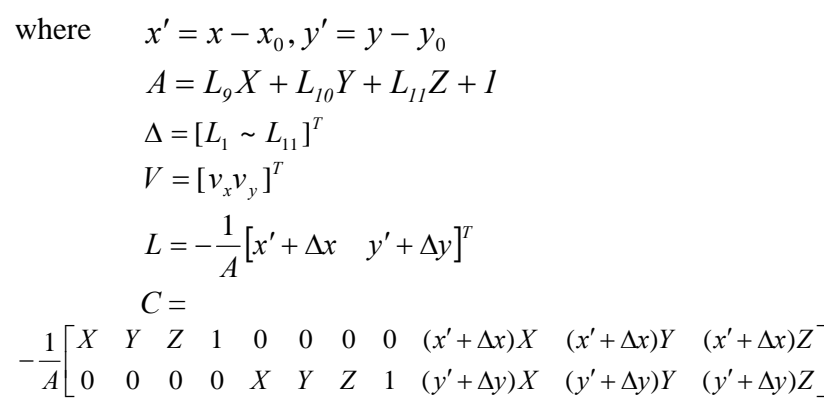

Normal equation is

$$
C^{T} C \Delta+C^{T} L=0
$$

3.3 Solution of the Distortion Parameters and elements of interior orientation

For weakening the influence of correlation between DLT coefficients and principal point of photograph, we regard $x_{0}$ and $y_{0}$ as undetermined parameters. From the Eq. (3) and the Eq. (4) we can obtain the following equation

$$
\left.\begin{array}{l}
-\left(k_{1} r^{2}+k_{2} r^{4}+k_{3} r^{6}\right) x^{\prime}-p_{1}\left(r^{2}+2 x^{\prime 2}\right)-2 p_{2} x^{\prime} y^{\prime} \\
+x_{0}-x-L_{x} / A=v_{x} \\
-\left(k_{1} r^{2}+k_{2} r^{4}+k_{3} r^{6}\right) y^{\prime}-2 p_{1} x^{\prime} y^{\prime}-p_{2}\left(r^{2}+2 y^{\prime 2}\right) \\
+y_{0}-y-L_{y} / A=v_{y}
\end{array}\right\}
$$

Type of Matrix is

where

$$
V=C \Delta+L
$$

$$
\begin{aligned}
C=- & -\left[\begin{array}{ccccccc}
r^{2} x^{\prime} & r^{4} x^{\prime} & r^{6} x^{\prime} & r^{2}+2 x^{\prime 2} & 2 x^{\prime} y^{\prime} & -1 & 0 \\
r^{2} y^{\prime} & r^{4} y^{\prime} & r^{6} y^{\prime} & 2 x^{\prime} y^{\prime} & r^{2}+2 y^{\prime 2} & 0 & -1
\end{array}\right] \\
& \Delta=\left[\begin{array}{lllllll}
k_{1} & k_{2} & k_{3} & p_{1} & p_{2} & x_{0} & y_{0}
\end{array}\right]^{T} \\
& V=\left[\begin{array}{lll}
v_{x} v_{y}
\end{array}\right]^{T} \\
& L=-\left[\begin{array}{lll}
x+L_{x} / A & y+L_{y} / A
\end{array}\right]^{T} \\
& L_{x}=L_{1} X+L_{2} Y+L_{3} Z+L_{4} \\
& L_{y}=L_{5} X+L_{6} Y+L_{7} Z+L_{8}
\end{aligned}
$$

Normal equation is

$$
C^{T} C \Delta+C^{T} L=0
$$

\section{CALIBRATION ALGORITHM OF MULTI-IMAGE RESECTION}

\subsection{Single Image Resection with Distortion Correction}

Because the collinear equation is non-linear transformations, the collinear equation can be linearized for solving orientation parameters. The linearization considers the Taylor series firstorder expansion of collinear equation collinear equation at the Initial value. The linearized collinear equation with distortion error correction (Chen Xingfeng, 2011b) is

$$
\begin{gathered}
{\left[\begin{array}{llllll}
c_{11} & c_{12} & c_{13} & c_{14} & c_{15} & c_{16} \\
c_{21} & c_{22} & c_{23} & c_{24} & c_{25} & c_{26}
\end{array}\right]\left[\begin{array}{l}
d X_{S} \\
d Y_{S} \\
d Z_{S} \\
d \varphi_{S} \\
d \omega_{S} \\
d \kappa_{S}
\end{array}\right]+\left[\begin{array}{lll}
c_{17} & c_{18} & c_{19} \\
c_{27} & c_{28} & c_{29}
\end{array}\right]\left[\begin{array}{l}
d f \\
d x_{0} \\
d y_{0}
\end{array}\right] \ldots} \\
+\left[\begin{array}{lllll}
d_{11} & d_{12} & d_{13} & d_{14} & d_{15} \\
d_{21} & d_{22} & d_{23} & d_{24} & d_{25}
\end{array}\right]\left[\begin{array}{l}
k_{1} \\
k_{2} \\
k_{3} \\
p_{1} \\
p_{2}
\end{array}\right]-\left[\begin{array}{l}
l_{x} \\
l_{y}
\end{array}\right]=0
\end{gathered}
$$

where $\quad X s, Y s, Z s, \varphi, \omega, \kappa=$ elements of exterior orientation $f, x_{0}, y_{0}=$ elements of interior orientation $k_{1}, k_{2}, k_{3}, p_{1}, p_{2}=$ distortion parameters

$$
\left\{\begin{array} { l } 
{ d _ { 1 1 } = ( x - x _ { 0 } ) r ^ { 2 } } \\
{ d _ { 1 2 } = ( x - x _ { 0 } ) r ^ { 4 } } \\
{ d _ { 1 3 } = ( x - x _ { 0 } ) r ^ { 6 } } \\
{ d _ { 1 4 } = r ^ { 2 } + 2 ( x - x _ { 0 } ) ^ { 2 } } \\
{ d _ { 1 5 } = 2 ( x - x _ { 0 } ) ( y - y _ { 0 } ) }
\end{array} \quad \left\{\begin{array}{l}
d_{21}=\left(y-y_{0}\right) r^{2} \\
d_{22}=\left(y-y_{0}\right) r^{4} \\
d_{23}=\left(y-y_{0}\right) r^{6} \\
d_{24}=2\left(x-x_{0}\right)\left(y-y_{0}\right) \\
d_{25}=r^{2}+2\left(y-y_{0}\right)^{2}
\end{array}\right.\right.
$$

$\left\{\begin{array}{l}c_{11}=\frac{1}{\bar{Z}}\left(a_{1} f+a_{3} x\right) \\ c_{12}=\frac{1}{\bar{Z}}\left(b_{1} f+b_{3} x\right) \\ c_{13}=\frac{1}{\bar{Z}}\left(c_{1} f+c_{3} x\right) \\ c_{14}=b_{1} \frac{x y}{f}-b_{2}\left(f+\frac{x^{2}}{f}\right)-b_{3} y \\ c_{15}=-\frac{x^{2}}{f} \sin \kappa-\frac{x y}{f} \cos \kappa-f \sin \kappa \\ c_{16}=y \\ c_{17}=\frac{x}{f} \\ c_{18}=1 \\ c_{19}=0 \\ l_{x}=x-x^{\prime}\end{array}\left\{\begin{array}{l}c_{21}=\frac{1}{\bar{Z}}\left(a_{2} f+a_{3} y\right) \\ c_{22}=\frac{1}{\bar{Z}}\left(b_{2} f+b_{3} y\right) \\ c_{23}=\frac{1}{\bar{Z}}\left(c_{2} f+c_{3} y\right) \\ c_{24}=b_{1}\left(f+\frac{y^{2}}{f}\right)-b_{2} \frac{x y}{f}+b_{3} x \\ c_{25}=-\frac{x y}{f} \sin \kappa-\frac{y^{2}}{f} \cos \kappa-f \cos \kappa \\ c_{26}=-x \\ c_{27}=\frac{y}{f} \\ c_{28}=0 \\ c_{29}=1 \\ l_{y}=y-y^{\prime}\end{array}\right.\right.$

From the Eq. (10) we can obtain the following observation equation

$$
\left\{\begin{array}{l}
v_{x}=c_{11} d X_{S}+c_{12} d Y_{S}+c_{13} d Z_{S}+c_{14} d \varphi+c_{15} d \omega+c_{16} d \kappa+c_{17} d f \\
+c_{18} d x_{0}+c_{19} d y_{0}+d_{11} k_{1}+d_{12} k_{2}+d_{13} k_{3}+d_{14} p_{1}-d_{15} p_{2}-l x \\
v_{y}=c_{21} d X_{S}+c_{22} d Y_{S}+c_{23} d Z_{S}+c_{24} d \varphi+c_{25} d \omega+c_{26} d \kappa+c_{27} d f \\
+c_{28} d x_{0}+c_{29} d y_{0}+d_{21} k_{1}+d_{22} k_{2}+d_{23} k_{3}+d_{24} p_{1}-d_{25} p_{2}-l y
\end{array}\right.
$$

Type of Matrix is

$$
V=C \Delta+L
$$

$$
\begin{aligned}
\text { where } & V=\left[v_{x} v_{y}\right]^{T}, L=-\left[\begin{array}{lllllllllll}
l_{x} & l_{y}
\end{array}\right]^{T} \\
C & =\left[\begin{array}{llllllllllllllll}
c_{11} & c_{12} & c_{13} & c_{14} & c_{15} & c_{16} & c_{17} & c_{18} & c_{19} & d_{11} & d_{12} & d_{13} & d_{14} & d_{15} \\
c_{21} & c_{22} & c_{23} & c_{24} & c_{25} & c_{26} & c_{27} & c_{28} & c_{29} & d_{21} & d_{22} & d_{23} & d_{24} & d_{25}
\end{array}\right] \\
\Delta & =\left[\begin{array}{lllllllllllllll}
d X_{S} & d Y_{S} & d Z_{S} & d \varphi & d \omega & d \kappa & f & x_{0} & y_{0} & k_{1} & k_{2} & k_{3} & p_{1} & p_{2}
\end{array}\right]^{T}
\end{aligned}
$$

Normal equation is

$$
C^{T} C \Delta+C^{T} L=0
$$




\subsection{Multi-Image Resection with Distortion Correction}

Since the single image resection using only one photo and fewer geometric constraints and fewer observed values to solve the parameters, furthermore the outdoor calibration portable cage is too small to cover the whole image even only half image, it is not easy to achieve the optimal solution. The interior orientation elements and the exterior orientation elements and the distortion error parameters are strongly correlated, so that the stability and reliability of calibration results become poor. However the solving accuracy of multi-image resection is better than the single image resection. The most important difference between them is taking many images of calibration portable cage in photo's different position. They have the same interior orientation elements and distortion error parameters, but the different exterior orientation elements. This is equivalent to increase not only redundant observation, but also greatly reduce the influence of correlation between the interior orientation elements and exterior orientation elements, but the negative effects are unknown parameters of error equation rapidly increase as the number of images, so that the works of calculation become too large and the higher precision initial values. Otherwise the iterative calculation time become longer, even not converge.

The basic resection algorithm of single image is the same as multi-image. The only difference is that used more than one photo to solve the same interior orientation elements and distortion error parameters. Let the number of images equal to $n$ and the number of GCPs is $m$. we can obtain the following observation equation

$$
A E+B F+D G-L=0
$$

where $A_{i}=$ coefficient matrix of exterior orientation $B_{i}=$ coefficient matrix of interior orientation $D_{i}=$ coefficient matrix of distortion parameters $E_{i}=\left[\begin{array}{llllll}d X_{i S} & d Y_{i S} & d Z_{i S} & d \varphi_{i} & d \omega_{i} & d \kappa_{i}\end{array}\right]^{T}$$$
F=\left[\begin{array}{lll}
d f & d x_{0} & d y_{0}
\end{array}\right]^{T}
$$$$
G=\left[\begin{array}{lllll}
k_{1} & k_{2} & k_{3} & p_{1} & p_{2}
\end{array}\right]^{T}
$$

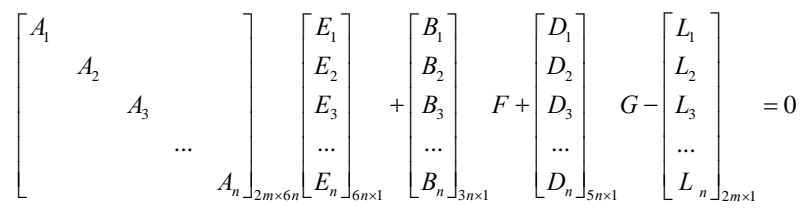

observation equation is

$$
\left\{\begin{array}{l}
V_{1}=A_{1} E_{1}+B_{1} F+D_{1} G-L_{1} \\
V_{2}=A_{2} E_{2}+B_{2} F+D_{2} G-L_{2} \\
V_{3}=A_{3} E_{3}+B_{3} F+D_{3} G-L_{3} \\
\cdots \\
V_{n}=A_{n} E_{n}+B_{n} F+D_{n} G-L_{n}
\end{array}\right.
$$

That is

$$
V_{2 m}=\left[\begin{array}{ccccccc}
A_{1} & 0 & 0 & 0 & 0 & B_{1} & D_{1} \\
0 & A_{2} & 0 & 0 & 0 & B_{2} & D_{2} \\
0 & 0 & A_{3} & 0 & 0 & B_{3} & D_{3} \\
& & & \cdots & & \cdots & \cdots \\
0 & 0 & 0 & 0 & A_{n} & B_{n} & D_{n}
\end{array}\right]_{2 m \times(6 n+8)}\left[\begin{array}{c}
E_{1} \\
E_{2} \\
E_{3} \\
\cdots \\
E_{n} \\
F \\
G
\end{array}\right]_{(6 n+8) \times 1}-L_{2 m}
$$

There are $6 n+8$ variables, so at least $3 n+4$ GCPs are need to solve Eq.(16). Type of Matrix is

$$
V=C \Delta+L
$$

\section{THE RESULTS OF EXPERIMENT}

This experiment used a Manfrotto tripod and the two Nikon D90 digital cameras with NIKKOR AF-S DX lens in each, image size: $23.6 \mathrm{~mm} \times 15.8 \mathrm{~mm}$, effective pixels: $4288 \times 2848$, focal length: $36 \mathrm{~mm}$, pixel size: $0.0055 \mathrm{~mm}$. e.g. Figure 2

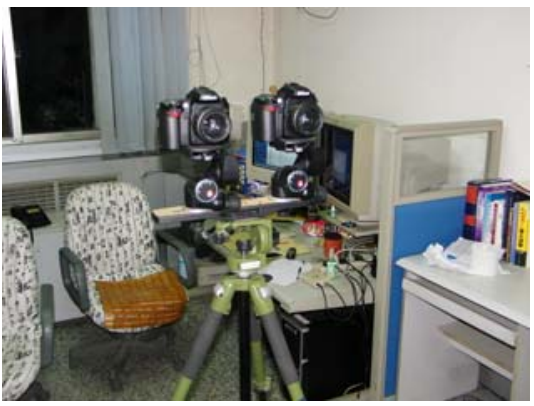

Figure 2 experiment devices

To prevent the small change of focal length to influence interior orientation element stability, we had fixed focal length in the experiment. In order to compare the experimental results, we used multi-image resection and DLT algorithm to solve interior orientation and the distortion parameter respectively. Both of them used the data of outdoor portable calibration steel cage.

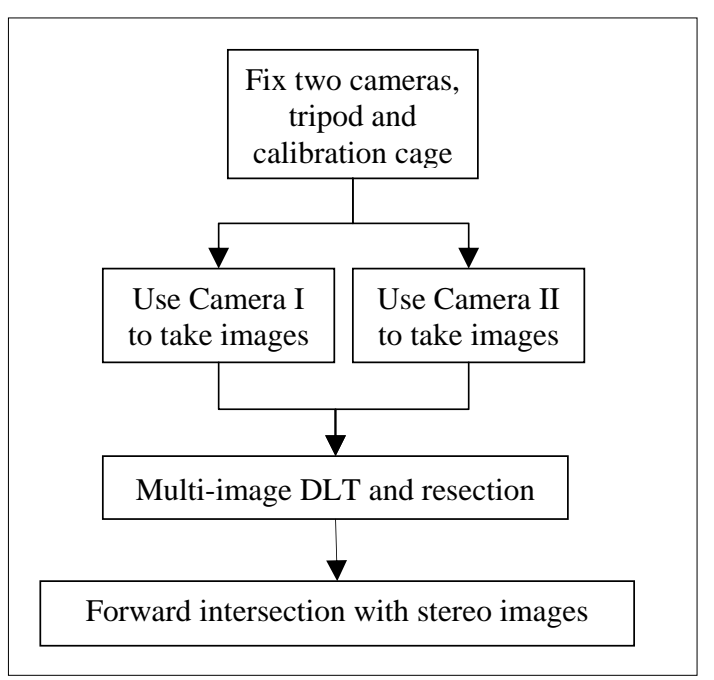

Figure 2 Standard Portable Steel Cage

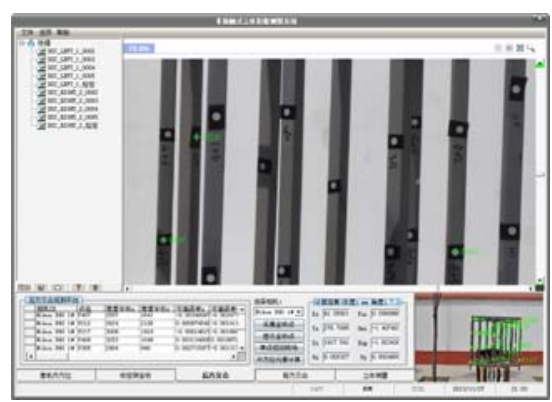

Figure3 UI of Calibration Software 
The calibration experimental was done in two steps, e.g. Figure 2. The first step, after two cameras and a tripod and a calibration cage were fixed, many multi-angle pictures were taken to solve each camera's interior orientation elements and distortion parameters, with multi-image DLT and resection independently. Table 1 gave the result of calibration and comparison.

\begin{tabular}{|c|c|c|c|c|}
\hline & \multicolumn{2}{|c|}{ DLT } & \multicolumn{2}{c|}{ Resection } \\
\hline & Camera I & Camera II & Camera I & Camera II \\
& $m m$ & $m m$ & $m m$ & $m m$ \\
$f$ & 36.134391 & 36.074060 & 36.3144857 & 36.3802218 \\
$x_{0}$ & 0.377716 & -0.029422 & 0.09867725 & 0.16534004 \\
$y_{0}$ & 0.132797 & 0.128374 & 0.04919723 & -0.14494807 \\
$k_{1}$ & $1.209 \mathrm{E}-004$ & $2.212 \mathrm{E}-004$ & $1.5803 \mathrm{E}-04$ & $1.5203 \mathrm{E}-04$ \\
$k_{2}$ & $9.149 \mathrm{E}-007$ & $-7.742 \mathrm{E}-007$ & $2.2004 \mathrm{E}-07$ & $1.1079 \mathrm{E}-07$ \\
$k_{3}$ & $-8.902 \mathrm{e}-009$ & $5.002 \mathrm{e}-010$ & $6.7031 \mathrm{E}-10$ & $2.31 \mathrm{E}-10$ \\
$p_{1}$ & $-7.787 \mathrm{e}-005$ & $-8.151 \mathrm{e}-005$ & $4.8981 \mathrm{E}-06$ & $2.7895 \mathrm{E}-05$ \\
$P_{2}$ & $2.016 \mathrm{e}-005$ & $-2.450 \mathrm{e}-005$ & $2.1424 \mathrm{E}-06$ & $1.7463 \mathrm{E}-05$ \\
\hline
\end{tabular}

Table 1. The Result of Interior Orientation and Distortion Parameters

The second step, a pair of stereoscopic images were used to compare the accuracy of forward intersection. The resection result of this stereoscopic pair was showed in table 2 and the forward intersection's result of stereoscopic pair was showed in Table 3.

\begin{tabular}{|c|c|c|c|c|}
\hline & \multicolumn{2}{|c|}{ DLT } & \multicolumn{2}{c|}{ Resection } \\
\hline & Camera I & Camera II & Camera I & Camera II \\
& $m m$ & $m m$ & $m m$ & $m m$ \\
$X_{S}$ & 91.0592 & 439.4953 & 89.7228 & 439.5092 \\
$Y_{S}$ & 1917.5416 & 1918.3010 & 1914.8721 & 1918.4376 \\
$Z_{S}$ & 276.7085 & 265.5823 & 276.7437 & 265.7132 \\
$\varphi$ & 0.2568 & 3.2346 & 0.2929 & 3.2381 \\
$\omega$ & 1.4074 & 0.1787 & 1.4107 & 0.1758 \\
$\kappa$ & 1.8234 & 2.0439 & 1.8200 & 2.0437 \\
MSE $x$ & 0.0023 & 0.0026 & 0.0037 & 0.0027 \\
MSE $y$ & 0.0024 & 0.0038 & 0.0043 & 0.0040 \\
\hline
\end{tabular}

Table 2. The Result of Multi-Image Resection

\begin{tabular}{|c|c|c|c|c|}
\hline \multirow{2}{*}{ GCP } & & $\begin{array}{c}X \\
\mathrm{~mm}\end{array}$ & $\begin{array}{c}Y \\
\mathrm{~mm}\end{array}$ & $\begin{array}{c}Z \\
\mathrm{~mm}\end{array}$ \\
\hline \multicolumn{5}{|c|}{ DLT } \\
\hline & MV $^{* 1}$ & 258.0880 & -4.0185 & 257.3924 \\
& $\mathrm{TV}^{* 2}$ & 257.8295 & -3.3466 & 257.3611 \\
& error & 0.2585 & -0.6719 & 0.0313 \\
& MV & 250.8088 & -485.2394 & 236.1824 \\
P2 & TV & 250.7558 & -485.83 & 236.3143 \\
& error & 0.0530 & 0.5906 & 0.1319 \\
& MV & 189.2726 & -178.0339 & 380.8347 \\
P3 & TV & 189.0691 & -177.5812 & 380.7502 \\
& error & 0.2035 & -0.4527 & 0.0845 \\
\hline \multicolumn{5}{|c|}{ Resection } \\
\hline P1 & MV & 257.7273 & -3.6918 & 257.3987
\end{tabular}

\begin{tabular}{|l|c|c|c|c|} 
& TV & 257.8295 & -3.3466 & 257.3611 \\
& error & -0.0102 & -0.3452 & 0.0376 \\
& MV & 250.5644 & -486.8108 & 236.3273 \\
& TV & 250.7558 & -485.83 & 236.3143 \\
& error & -0.1914 & -0.9808 & 0.013 \\
& MV & 189.0563 & -178.4958 & 380.6049 \\
& TV & 189.0691 & -177.5812 & 380.7502 \\
& error & -0.0128 & -0.9146 & -0.1453 \\
\hline
\end{tabular}

*1 measured value

*2 true value

Table 3. The Result of Forward Intersection

\section{CONCLUSION}

The results of accuracy show that the maximum $\mathrm{X}$ error was $0.2585 \mathrm{~mm}$, the maximum $\mathrm{Y}$ error was $0.6719 \mathrm{~mm}$ and the maximum $\mathrm{Z}$ error was $0.1319 \mathrm{~mm}$ by using multi-image DLT algorithm. On the other hand, the maximum $X$ error was $0.1914 \mathrm{~mm}$, the maximum $\mathrm{Y}$ error was $0.9808 \mathrm{~mm}$ and the maximum $\mathrm{Z}$ error was $0.1453 \mathrm{~mm}$ by using multi-image resection algorithm.

The forward intersection accuracy of the two methods was quite, and the both were less than $1 \mathrm{~mm}$. By using multi-image DLT algorithm the planimetric accuracy was less than $0.2585 \mathrm{~mm}$ and the height accuracy was less than $0.6719 \mathrm{~mm}$. On the other hand by using multi-image resection algorithm the planimetric accuracy was less than $0.1914 \mathrm{~mm}$ and the height accuracy was less than $0.9808 \mathrm{~mm}$. The planimetric accuracy of resection algorithm was the better than DLT algorithm, but the elevation accuracy of DLT algorithm was the better than resection algorithm.

In summary both method can be accepted for non-metric camera calibration. But also the solver accuracy in the inner orientation elements and distortion parameters was not very high has been noted. However for non-metric camera, the true value of inner orientation elements and lens distortion were unknown did not affect the accuracy of photogrammetry.

\section{REFERENCES}

Wang Lei, J., 2002. Master Thesis, Application and research of digital close-range photography, In: Institute of Surveying and Mapping, Information Engineering University,.Zhengzhou, China,pp 35-48.

Miao Hongjie, J., 2005. Discussions on the issues in calibration of digital cameras and photogrammetry. Journal of Capital Normal University(Natural Science dition), 36(1), pp. 117-120.

HE Min, J., 2011a. The tesearch on several methods of camera calibration, Journal of Geomatics \& Spatial Information Technology, 34(6), pp. 275-277.

Shao Xihui, J., 1991. M. engineer photogrammetry. Institute of Surveying and Mapping press, Zhengzhou, China, pp. 76-122.

Chen Xingfeng, J., 2011b. Multi-Image space resection based geometric calibration for the four band CCD camera, Journal of Remote Sensing For Land \& Resources, 1, pp 21-24. 